\title{
Causes, Human Health Impacts and Control of Harmful Algal Blooms: A Comprehensive Review
}

\author{
Sangeeta Sonak ${ }^{\mathrm{a}}$, Kavita Patil ${ }^{\mathrm{b}}$, Prabha Devi ${ }^{\mathrm{c}}$, Lisette D'Souza ${ }^{\mathrm{c}}$ \\ ${ }^{a}$ Centre for Environment and Natural Resource Management, F-4, Models Exotica, St Inez, Panaji- Goa, 403001, \\ India \\ ${ }^{\mathrm{b}}$ The Energy and Resources Institute, Alto-St Cruz, Bambolim, Goa, 403 202, India \\ ${ }^{\mathrm{c}}$ Bioorganic Chemistry Lab, National Institute of Oceanography, CSIR, Dona Paula, Goa, India \\ Email: sangeeta.sonak@gmail.com
}

\begin{abstract}
Harmful algal blooms (HABs) are increasingly attracting attention all over the world. A diverse set of algal species including diatoms, flagellates, chrysophytes and dinoflagellates can cause harmful blooms, and many produce toxins that harm other organisms and human health. Intensive cyanobacterial blooms, in particular, have been associated with high costs for society due to their potential toxicity. Algal blooms can produce different toxins. These toxins present a long-standing threat to human and environmental health. For example, the severe Karenia bloom of 2005 in the eastern Gulf of Mexico demonstrates the complexities and magnitude of the challenges of managing algal blooms for both environmental and public health. With increasing events and severe impacts of algal blooms on human health, it becomes necessary to monitor and manage toxic algal blooms. Hence this paper presents a review of causes, impacts and control of algal blooms.
\end{abstract}

\section{Introduction}

Harmful algal blooms (HABs) are increasingly attracting attention all over the world. The dynamics of rapid increase or almost equally, decrease of phytoplankton populations is known as a 'bloom'[1]. The term harmful covers those microalgal species, which can cause damage to marine living resources and ecosystems and create a negative impact on human welfare[2]. A harmful algal bloom (HAB) is thus defined as a bloom that has deleterious effects on plants, animals and/or humans[3]. Low nitrogen/phosphorus ratios and increased temperatures are known to be the most critical factors for increasing events of harmful algal blooms $[4,5]$.

Algal blooms can produce different toxins. A diverse set of algal species including diatoms, flagellates, chrysophytes and dinoflagellates can cause harmful blooms, and many produce toxins that harm other organisms and human health. Intensive cyanobacterial blooms, in particular, have been associated with high costs to society due to their potential toxicity, with associated negative effects on recreational waters and on water supplies used for the preparation of drinking water [4]. With increasing events and severe impacts of algal blooms on human health, it becomes necessary to monitor and manage toxic algal blooms. Hence this paper presents a review of causes, impacts and control of algal blooms.

\section{Causes of HABs}

Unfortunately, the causes of HABs are uncertain to date. There is no conclusive report available for the causes of HABs. However, some of the factors which are thought to be responsible for causing HABs are succinctly described below.

\subsection{Coastal Eutrophication}

Coastal pollution resulting from various sources like domestic and industrial effluents is one of the most important factors in the development of HABs. On most occasions, eutrophication due to nutrient enrichment results in algal blooms, some of which are toxic to marine organisms and to humans. Recent research suggests that eutrophication and climate change are two processes that may promote the proliferation and expansion of cyanobacterial blooms[6]. Eutrophication or nutrient enrichment can alter 
the species composition of an ecosystem[5]. Native biota may be displaced as the environment becomes enriched with nutrients such as nitrates and phosphates[7]. Coastal eutrophication and enhanced offshore nutrient concentrations occurring offshore due to vertical mixing have been linked with the development of large biomass, ultimately leading to anoxia, toxic or harmful impacts on ecosystems, human health or recreation and on fisheries resources. As eutrophication increases nitrogen and phosphorus inputs, the ratio of these nutrients to silicates becomes very high. This favours non-diatom species including several harmful species. There are some cases like that of the Black Sea, where an increase in eutrophication accompanied by a reduction in silicate concentrations has led to the proliferation of flagellates including diatom blooms[8]. Further, it is believed that high concentration of phosphorus, and a low total nitrogen to total phosphorus (TN:TP) TN: TP ratio, are favorable for the production of cyanobacteria blooms. According to the several studies, cyanobacteria usually dominate in lakes with low TN/TP ratio and are rare in lakes with high TN:TP ratios[9,10]. Cyanobacteria dominate in lakes where TN:TP mass ratio is below 29:1[10]. Lukatelich and McComb[11] found that summer blooms of the cyanobacterium Nodularia in the Harvey Estuary (Western Australia) were directly related to total riverine phosphorus loadings during the previous winter. Preece et al. [12] in their recent research also agree that toxin production by harmful cyanobacteria blooms constitutes a major, worldwide environmental threat to freshwater aquatic resources that is expected to expand in scale and intensity with global climate change. However, they further strongly suggest that increased monitoring and research efforts to understand, react to, and prevent ecological and health problems associated with the growing problem of toxic CyanoHABs in coastal environments are required[12]. They highlight a potential food-borne exposure route to humans by reviewing the growing body of evidence that shows microcystins can accumulate in coastal seafood in order to substantiate their arguments.

Nutrients derived from anthropogenic activities have resulted in the increase in HAB records. In some places, unusual heavy rains have resulted in blooms of L. polyedrum due to nutrient rich runoff into the coastal waters e.g. Santa Barbara to south of Mexican border[13]. Rainfall being one of the nutrient resources and in; places like Tolo bay, Hong Kong and the Seto Inland Sea in Japan have resulted in increased red tide episodes with increase in eutrophication[8]. Japanese waters also detected presence of new harmful species e.g. Heterocapsa circularisquama[8]. Blooms of dinoflagellate Pfiesteria are reported in estuaries of middle and southern Atlantic coasts. Water salinity, pH, nutrients and temperature are the main factors controlling cycles of dinoflagellates, but apart from these, studies in North California have illustrated that they thrive well near sources of organic phosphates released from sewage treatment plants[14]. According to Garcia-Hernandez et. al.[15], increased aquaculture activities like that of shrimp farming in Kun Kaak Bay, along coastal Sonora in Mexico, have resulted in a drastic change from blooms of diatoms to dinoflagellates and then Rhaphidophytes, which are again well-known indicators of eutrophication. This may be related to untreated nutrient-rich effluents discharged into Kun Kaak bay.

Similarly, Semeneh et. al.[16]studied the nitrogen uptake regime in relation to the biomass and structures of the phytoplankton community in the Atlantic and Indian sectors of the Southern Ocean. Southern Oceans fall in the category of High Nutrient Low Chlorophyll (HNLC) trophic category showing very low utilization of nutrients. In some of these parts, the net removal of silicate during the growth season exceeds that of nitrate and cycling of $\mathrm{N}$ as $\mathrm{NH}_{3}$ leads to uncoupling of $\mathrm{Si}$ and $\mathrm{N}$ cycles on the surface of the water. As discussed by Semeneh et. al.[16], in the Coastal and Continental Shelf Zone (CCSZ) and Open Oceanic Zone (OOZ) of the Indian sector, a bloom can develop only when the rate of biomass increase exceeds the loss rate i.e. grazing rate and sedimentation rate. Once a bloom develops, it persists for a longer period under low growth rate if the loss rate is small. While the interactive effects of future eutrophication and climate change on harmful algal blooms are complex, much of the current knowledge suggests these processes are likely to enhance the magnitude and frequency of these events[6].

\subsection{Dust Storms and Metals}

Satellite studies carried out by the University of South Florida's College of Marine Science found that giant dust clouds that blow across the Atlantic Ocean from the Sahara Desert towards the Gulf of Mexico are responsible for red tides. Data revealed that these clouds fertilize the water off the West Florida Coast with iron. Cyanobacteria like species of Trichodesmium use Fe content from dust particles to fix atmospheric nitrogen in coastal water making the Gulf of Mexico a productive environment for toxic algae thus causing red tides[17]. Further studies showed that there was an increase in Fe content 
in surface waters by $300 \%$, thereby increasing the nitrogen content in this water. This change resulted in bloom of the toxic red alga Karenia brevis between the regions of Tampa Bay and Fort Myers, Florida[17].

Albert et al.[18] documented some common factors responsible for blooms of the cyanobacterium Lyngbya majuscula at coastal Queensland in Australia. Runoff during rain events introduces iron, phosphorus and dissolved organics into the coastal zone. A combination of iron and phosphorus is required to increase the photosynthetic activity of L. majuscule, thus causing a bloom. Fe plays a very important role in nitrogen acquisition. An increase in $\mathrm{Fe}$ may initiate blooms of Aureococcus anophagefferens resulting in brown tides. One good example is that of Peconic estuary, New York, USA[19]. Further, studies of Kun Kaak Bay, Mexico, by Garcia-Hernandez et. al.[15] have revealed higher concentrations of heavy metals such as $\mathrm{Cd}$ and $\mathrm{Pb}$ due to untreated nutrient-rich effluents from aquacultural activities, which are frequent along the Sonora coastline. This could be one good example for increase of metals in ocean due to aquacultural activities leading to HABs.

\subsection{Climate Change and Increase in Temperature}

According to Peperzak[20], climate change is one of the factors for HAB in the Dutch costal zone of the North Sea. Increase in temperature and in precipitation associated with climate change fall into wide ranges, and uncertainties exist in their effect on stratification and North Sea flushing. Dinoflagellates and raphidophytes increase considerably. Species of D. acuminata, P. minimum, F. japonica and $C$. antiqua are observed in this region regularly, indicating an increase in $\mathrm{HAB}$ [20]. Temperature variation affects circulation patterns, prolongs stratification periods and causes variations in the physical structure of water column that favours occurrence of $\operatorname{HABs}[8]$.

\subsection{Upwelling of Oceans}

Ascending or vertical motions caused due to oceanic circulation is known as 'Upwelling', which affects the environmental conditions, increases the nutrient content in euphotic zone thereby increasing the productivity of the region[21]. Sharma[21] has discussed various factors inducing upwelling off the south west coast of India. Further, according to Garcia-Hernandez et. al.[15], Kun Kaak Bay located in the south of Seri or Comcaac territory and the northwest of the fishing town of Kino bay in northern Sonora is known for its high productivity resulting from seasonal upwelling currents. Here, diatom blooms (e.g. Stephanopyxis palmeriana) were common in the past. Similarly, in the Gulf of California, non-toxic red tide algal blooms are common. Mesodinium rubrum is a common red tide species associated with upwelling in these areas. Ramana and Reddy[22] have carried out studies to find out the occurrence of the upwelling process along the Dakshina Kannada Coast of India and they report that upwelling was found to occur from March to October along the coast. This could be one of the factors for HABs along the southwest coast of India.

\subsection{Ballast Water}

Though there is limited research done in terms of the spread of harmful algal species through ballast water, according to Anil et al. [23], ballast water is considered to be one of the important vectors for spread of HAB species. For example, Untawale et. al.[24] documented that algal species like Monostroma oxysporum, which are native of northeast Atlantic and northwest Pacific, were introduced to the West Coast of India. Anil et al. [23], further suggest that India being one of the major maritime countries is susceptible to such type of bioinvasion from the rest of the world oceans and thus requires detailed research in terms of spread of HAB-causing species.

\subsection{Unhealthy Coral Reefs}

Unhealthy coral reefs play a very important role in the formation of blooms. According to Waldichuk[25], healthy coral reefs are free of external algal growth. Unhealthy conditions or death of corals due to oil pollution or depositions of sediments lead to encrustations of corals by calcareous materials and algae, and may in turn lead to the death of zooplanktons or higher fishes in the food web. 
Endolithic algal bloom causes a disease called White Syndrome (WS), consisting of distinct lines between healthy corals and dead ones. These endolithic algae (primarily Ostreobium spp.) penetrate the coral tissues of tabular Acropora spp., which in turn may affect the corals leading to micro-lessions, and making them susceptible to infiltration by potential pathogens. One good example is that of Gambierdiscus toxicus, a benthic dinoflagellate that finds its way on dead corals. G. toxicus releases ciguatoxin thus causing Ciguatera Fish Poisoning (CFP). Thus Waldichuk[25] concludes that if a contaminant or a development affects water quality to such an extent that coral reefs are affected than G.toxicus is likely to bloom, causing extensive release of ciguatoxin.

\section{Impacts of $\mathrm{HAB}$}

Harmful cyanobacterial blooms (cHABs) have significant socioeconomic and ecological costs, which impact drinking water, fisheries, agriculture, tourism, real estate, water quality, food web resilience and habitats, and contribute to anoxia and fish kills[26]. High biomass accumulation and degradation of algal blooms may lead to depletion of dissolved oxygen, light attenuation, clogging of fish gills etc. These effects can result in fish kills involving thousands of fish and other marine life, and lead to degradation of the ecosystem[27]. Nevertheless, the most critical impacts of algal blooms are on human health, which will be discussed in detail in this section. Worldwide HABs that produce toxins, which cause acute or chronic health effects in mammals (including humans) and other organisms[26].Toxins produced during harmful algal blooms are some of the most powerful natural toxic substances known. Toxins can directly kill fish or shellfish or other marine life or may accumulate in fish and sea food and lead to human poisoning after ingestion of contaminated sea food[3,27].

\subsection{Human Health Impacts}

As explained above, toxins produced during algal blooms may not be toxic to fish and marine life. However, they accumulate in fish and mollusks and move up the food chain to have a devastating impact on humans. Marine toxin diseases are categorized into two types based on their trans-vectors. Shellfish carry toxins that lead to paralytic shellfish poisoning, neurotoxic shellfish poisoning, diarrheic shellfish poisoning and amnesic shellfish poisoning. Poisoning through mollusks tends to occur during algal bloom episodes. Fish carry toxins that lead to ciguatera and tetrodotoxin poisoning. Fish poisoning is more localized and associated with parts of specific reefs or fish. Bloom Episodes of dinoflagellate Pfiesteria in estuaries of middle and Southern Atlantic coast suggest that anthropogenic stress on aquatic environment has caused fish kills and related health hazards in humans ${ }^{14}$. Species of Pfiesteria are also reported to cause lesions in fish.

Additionally, humans can be exposed to toxins that are directly released into water or air. This occurs naturally or as a result of cell disruption caused by turbulence or through human activities like water treatment. This phenomenon frequently occurs in the Gulf of Mexico where residents and beach goers are exposed to toxins through seas spray. Toxins can then be inhaled and lodged in the nose and throat or down into the lungs. Common symptoms associated with this are respiratory irritation, coughing, and other ailments[27]. Table 1 shows incidents of HABs described in the literature.

Table 1. Incidents of HABs described in the literature

\begin{tabular}{l|l|l|l|l}
\hline $\begin{array}{l}\text { Year of } \\
\text { Incident }\end{array}$ & $\begin{array}{l}\text { Geographical } \\
\text { Location }\end{array}$ & Causative Organisms & Impacts & Literature \\
\hline 1879 & $\begin{array}{l}\text { Western Gulf of } \\
\text { Mexico }\end{array}$ & Karenia brevis & $\begin{array}{l}\text { Human respiratory irritation was } \\
\text { first reported. }\end{array}$ & {$[28]$} \\
\hline $1900-1989$ & North America & $\begin{array}{l}\text { PSP (paralytic shellfish } \\
\text { poisoning) produced by } \\
\text { several species }\end{array}$ & $\begin{array}{l}1399 \text { people intoxicated and } 90 \\
\text { deaths }\end{array}$ & {$[29]$} \\
\hline $1901-1992$ & Norway & PSP outbreak & 32 people intoxicated and 2deaths & {$[29]$} \\
\hline 1917 & $\begin{array}{l}\text { Eastern Gulf of } \\
\text { Mexico }\end{array}$ & Karenia brevis & $\begin{array}{l}\text { Human respiratory irritation was } \\
\text { first reported. }\end{array}$ & {$[30]$} \\
\hline
\end{tabular}




\begin{tabular}{|c|c|c|c|c|}
\hline \multirow{2}{*}{$\begin{array}{l}\text { March } \\
1942\end{array}$} & \multirow[t]{2}{*}{ Lake Hamana, Japan } & \multirow[t]{2}{*}{ Prorocentrum. minimum } & $\begin{array}{l}114 \text { of } 324 \text { affected people died } \\
\text { after consuming oysters (Venerupis } \\
\text { semidecussata) and } \\
\text { short-necked clams (Tapes } \\
\text { semidecussata) }\end{array}$ & {$[31]$} \\
\hline & & & $\begin{array}{l}342 \text { people affected after } \\
\text { consumption of clams Tapes } \\
\text { japonica }\end{array}$ & {$[32-35]$} \\
\hline March 1943 & Lake Hamana, Japan & Prorocentrum. minimum & $\begin{array}{l}\text { Seventy-one deaths were attributed } \\
\text { to ingestion of toxic oysters } \\
\text { (Crassostrea gigas) from the same } \\
\text { region }\end{array}$ & {$[31]$} \\
\hline $\begin{array}{l}1946 / \\
1994 \\
\end{array}$ & Portugal & PSP outbreak & $\begin{array}{l}132 \text { people intoxicated and } 7 \\
\text { deaths }\end{array}$ & {$[29]$} \\
\hline 1947 & West coast of Florida & $\begin{array}{l}\text { Brevetoxins released by } \\
\text { red tide blooms }\end{array}$ & $\begin{array}{l}\text { Respiratory irritation due to } \\
\text { inhalation of aerosolized } \\
\text { brevetoxins. Seawater containing } \\
\text { red tide organisms was sprayed as } \\
\text { an aerosol into the nose and throat } \\
\text { of volunteers, coughing and } \\
\text { burning sensation }\end{array}$ & {$[36]$} \\
\hline March 1949 & Lake Hamana, Japan & Prorocentrum. minimum & $\begin{array}{l}\text { Ingestion of toxic clams. } \\
\text { Symptoms included heavy liver } \\
\text { injury (necrosis and fatty } \\
\text { degeneration), hemorrhage } \\
\text { diathesis with frenzy, } \\
\text { unconsciousness and coma, and } \\
\text { death occurring } 24-48 \mathrm{~h} \text { after } \\
\text { symptoms appeared } \\
\end{array}$ & {$[31]$} \\
\hline 1960 & Florida waters & Karenia brevis & $\begin{array}{l}\text { Human poisonings associated with } \\
\text { bivalve shellfish consumption }\end{array}$ & {$[37,38]$} \\
\hline 1968 & UK & PSP outbreak & 78 people intoxicated & {$[29]$} \\
\hline $\begin{array}{l}\text { November } \\
1972\end{array}$ & $\begin{array}{l}\text { East coast of Florida } \\
\text { throughout Palm } \\
\text { beach County }\end{array}$ & $\begin{array}{l}\text { K. brevis- inhalation of } \\
\text { aerosolized brevetoxins }\end{array}$ & $\begin{array}{l}\text { People on beach (swimmers, } \\
\text { workers, lifeguards) showed } \\
\text { symptoms of acute eye and nose } \\
\text { irritation, non-productive cough } \\
\text { and respiratory distress. }\end{array}$ & {$[39]$} \\
\hline $\begin{array}{l}1972 / \\
1997 \\
\end{array}$ & Chile & PSP outbreak & $\begin{array}{l}329 \text { people intoxicated and } 23 \\
\text { deaths }\end{array}$ & {$[29]$} \\
\hline 1976 & Malaysia & $\begin{array}{l}\text { Blooms of Pyrodinium } \\
\text { Bahamense var. } \\
\text { compressum, }\end{array}$ & $\begin{array}{l}\text { Affecting } 202 \text { people and causing } \\
\text { death of } 7 \text { children's. }\end{array}$ & {$[40]$} \\
\hline $\begin{array}{l}1976 ; \\
1977 ; 1987 ; \\
\text { May } 1993 \\
\end{array}$ & $\begin{array}{l}\text { Off Mangalore } \\
\text { Southwest coast of } \\
\text { India }\end{array}$ & $\begin{array}{l}\text { Blooms of Noctiluca } \\
\text { miliaris }\end{array}$ & Not Known & {$[41]$} \\
\hline 1976-1977 & Along Kerala coast & Noctiluca Miliaris & No impacts & {$[42]$} \\
\hline $1979-1981$ & Norway & Prorocentrum. Minimum & $\begin{array}{l}\text { Mussels consumption caused } \\
\text { human poisoning }\end{array}$ & {$[43,44]$} \\
\hline 1981 & Spain & DSP cases & 500 cases of DSP were reported & {$[45]$} \\
\hline 1983 & $\begin{array}{l}\text { Mangalore, KT in } \\
\text { India }\end{array}$ & Species not identified & $\begin{array}{l}\text { Several hospitalized due to } \\
\text { consumption of shell fish }\end{array}$ & {$[46]$} \\
\hline $1984 \& 1986$ & France & DSP cases & DSP 2000 cases & {$[45]$} \\
\hline
\end{tabular}




\begin{tabular}{|c|c|c|c|c|}
\hline $\begin{array}{l}\text { Since 1983, } \\
\text { 1998-1999 }\end{array}$ & $\begin{array}{l}\text { Philippines } \\
\text { Manila Bay and } \\
\text { Malampaya Sound }\end{array}$ & $\begin{array}{l}\text { Pyrodinium bahamense } \\
\text { var. compressum) }\end{array}$ & $\begin{array}{l}\text { Affects the shellfish industry but } \\
\text { also endangers the health of the } \\
\text { public through consumption of } \\
\text { shellfish contaminated with toxins. }\end{array}$ & {$[47]$} \\
\hline April 1985, & $\begin{array}{l}\text { Tadri Estuary in } \\
\text { India }\end{array}$ & \begin{tabular}{|l|} 
Toxic dinoflagellate was \\
not detected however PSP \\
was detected in oysters \\
\end{tabular} & Not Known & {$[48]$} \\
\hline $1985-1986$ & $\begin{array}{l}\text { Along the coast of } \\
\text { Karnataka in India }\end{array}$ & $\begin{array}{l}\text { Species of D.caudate-low } \\
\text { levels of PSP detected }\end{array}$ & $\begin{array}{l}\text { Caused DSP in infected shellfishes } \\
\text { collected from the harvesting areas }\end{array}$ & {$[48]$} \\
\hline 1985 & Long Island, USA, & Prorocentrum. Minimum & $\begin{array}{l}\text { Clams, Mercenaria mercenaria, } 3 \\
\text { humans affected }\end{array}$ & {$[49]$} \\
\hline 1987 & $\begin{array}{l}\text { Prince Edward } \\
\text { Island, Canada }\end{array}$ & $\begin{array}{l}\text { Diatom Pseudo-nitzschia } \\
\text { caused ASP }\end{array}$ & $\begin{array}{l}\text { Four Canadians lost their lives } \\
\text { when exposed to toxic mussels }\end{array}$ & {$[45]$} \\
\hline 1987 & Guatemala & PSP outbreak & $\begin{array}{l}187 \text { people intoxicated and } 26 \\
\text { deaths }\end{array}$ & {$[29]$} \\
\hline October 1987 & $\begin{array}{l}\text { North Carolina } \\
\text { Coast. }\end{array}$ & $\begin{array}{l}\text { Outbreak of NSP } \\
\text { secondary to red tide of } \\
\text { K. brevis ( then known as } \\
\text { P. brevis). }\end{array}$ & $\begin{array}{l}48 \text { persons were diagnosed with } \\
\text { NSP following consumption of } \\
\text { cooked and raw oysters at } 20 \\
\text { different meals. Acutely } 23 \% \text { of } \\
\text { cases reported gastrointestinal and } \\
39 \% \text { neurologic symptoms. } \\
\end{array}$ & {$[50]$} \\
\hline $\begin{array}{l}1988 \text { May- } \\
\text { June }\end{array}$ & $\begin{array}{l}\text { Kattegat and } \\
\text { Skagerrak area } \\
\text { (North Sea) } \\
\end{array}$ & $\begin{array}{l}\text { Blooms of } \\
\text { Chrysochromulina } \\
\text { Polylepis }\end{array}$ & $\begin{array}{l}\text { Caused death of } 900 \text { tonnes of fish, } \\
\text { including cod, salmon and trout }\end{array}$ & {$[8]$} \\
\hline 1990 & Denmark & $\begin{array}{l}\text { Mussels exported from } \\
\text { Denmark to France }\end{array}$ & DSP poisoning in over 400 people. & {$[45]$} \\
\hline $\begin{array}{l}\text { October and } \\
\text { November } \\
1991\end{array}$ & $\begin{array}{l}\text { Oregon and } \\
\text { Washington Coasts }\end{array}$ & $\begin{array}{l}\text { Organism unknown } \\
\text { DA (Domoic Acid) found } \\
\text { Razor clams (Siliqua } \\
\text { patula Dixon) and } \\
\text { Dungeness crabs (Cancer } \\
\text { magister Dana) } \\
\end{array}$ & $\begin{array}{l}\text { People became ill after consuming } \\
\text { razor clams }\end{array}$ & {$[13]$} \\
\hline $\begin{array}{l}1991 \text { and } \\
1992\end{array}$ & $\begin{array}{l}\text { Kattegat and } \\
\text { Skagerrak area } \\
\text { (North Sea) }\end{array}$ & Blooms of prymnesiophyte & Not Known & {$[8]$} \\
\hline 1992-1993 & New Zealand & $\begin{array}{l}\text { Karenia mikimotoi along } \\
\text { with suspect species in } \\
\text { bloom }\end{array}$ & $\begin{array}{l}\text { Outbreak of NSP due to } \\
\text { consumption of cockles and green } \\
\text { shell mussels; oysters }\end{array}$ & {$[51]$} \\
\hline 1994 & $\begin{array}{l}\text { Hood Canal of } \\
\text { Western Washington. }\end{array}$ & $\begin{array}{l}\text { Blooms of pseudo-nitzchia } \\
\text { spp. Mainly P.multiseries, } \\
\text { P.australis and P. pungens } \\
\text { persisted for more than } 6 \\
\text { weeks }\end{array}$ & $\begin{array}{l}\text { Mussels indicated the presence of } \\
\text { domoic acid however impacts are } \\
\text { not reported }\end{array}$ & {$[13]$} \\
\hline $1995-1996$ & West coast of Florida & Karenia brevis & $\begin{array}{l}\text { Eye and respiratory irritation. Six } \\
\text { person were hospitalized for illness } \\
\text { attributed to red tide exposure }\end{array}$ & {$[52]$} \\
\hline
\end{tabular}




\begin{tabular}{|c|c|c|c|c|}
\hline 1996-1997 & $\begin{array}{l}\text { In estuaries of eastern } \\
\text { shore of Maryland }\end{array}$ & $\begin{array}{l}\text { Episodes of dinoflagellate } \\
\text { Pfiesteria }\end{array}$ & $\begin{array}{l}\text { Anthropogenic stress on aquatic } \\
\text { environment have caused fish kills } \\
\text { and related health hazards in } \\
\text { humans - fatigue, headache, } \\
\text { respiratory problems, diarrhea, } \\
\text { weight loss, skin irritation and } \\
\text { memory difficulties }\end{array}$ & {$[14]$} \\
\hline 1997 & Kerala in India & $\begin{array}{l}\text { Paralytic shellfish } \\
\text { poisoning }\end{array}$ & $\begin{array}{l}\text { Seven deaths, over } 500 \text { hospitalized } \\
\text { after consuming mussels Perna } \\
\text { indica. }\end{array}$ & {$[46]$} \\
\hline$(1998-2006)$ & $\begin{array}{l}\text { Northwest Pacific } \\
\text { (NWP) } \\
\text { (Covering China, } \\
\text { Korea, Japan and } \\
\text { Russia) }\end{array}$ & $\begin{array}{l}\text { Diatoms, Dinoflagellates, } \\
\text { Ciliates and } \\
\text { Raphidophytes : } \\
\text { Noctilula scintillans } \\
\text { Skeletonema costatum } \\
\text { Ceratium furca, Karenia } \\
\text { digitata, Prorocentrum } \\
\text { dentatum, Karania } \\
\text { mikimotoi Mesodinium } \\
\text { rubrum,Cochlodinium } \\
\text { polykrikoides, } \\
\text { Alexandrium tamarense, } \\
\text { Alexandrium catenella, } \\
\text { Gymnodinium } \\
\text { mikimotoi,Heterosigma } \\
\text { akashiwo, Gymnodinium } \\
\text { catenatum, Exuviaella } \\
\text { marina, } \\
\text { Leptocylindrus danicus, } \\
\text { Karania mikimotoi, } \\
\text { Eucampia zoodiacus, } \\
\text { Mesodinium rubrum, } \\
\text { Skeletonema costatum } \\
\text { etc }\end{array}$ & $\begin{array}{l}\text { Summer algal blooms causing fish } \\
\text { mortality, shellfish poisoning, } \\
\text { physiological impairment, and } \\
\text { numerous ecological and health } \\
\text { impacts }\end{array}$ & {$[53]$} \\
\hline $\begin{array}{l}\text { February } \\
1999\end{array}$ & $\begin{array}{l}\text { Offshore red tide off } \\
\text { the Gulf coast of } \\
\text { Florida. }\end{array}$ & $\begin{array}{l}\text { Karenia brevis red tide } \\
\text { events, }\end{array}$ & $\begin{array}{l}\text { Data suggest that people can } \\
\text { experience upper and lower } \\
\text { respiratory irritation and some } \\
\text { inflammatory response after } \\
\text { inhaling aerosolized brevetoxins } \\
\text { during red tide events. }\end{array}$ & {$[54]$} \\
\hline $\begin{array}{l}2001 \text { and } \\
2006 \text {, }\end{array}$ & Cuba & Not known & $\begin{array}{l}\text { Symptoms of intoxication of } \\
\text { Ciguatera Fish Poisoning (CFP) } \\
\text { after consuming carangid ( Caranx } \\
\text { latus). Commonly called as "jurel" } \\
\text { in Cuba. } 570 \text { reported cases of fish } \\
\text { intoxication, } 72 \% \text { of which were } \\
\text { due to CFP }\end{array}$ & {$[55]$} \\
\hline
\end{tabular}




\begin{tabular}{|c|c|c|c|c|}
\hline $\begin{array}{l}2001 \\
\text { September - } \\
\text { December } \\
2002 \text { same } \\
\text { months } \\
\end{array}$ & Florida waters, USA & $\begin{array}{l}\text { Karenia brevis red tides } \\
\text { exposure period } \\
\text { No red tide bloom } \\
\text { occurred }\end{array}$ & $\begin{array}{l}\text { People located near the coast } \\
\text { reflected higher or more chronic } \\
\text { exposure to Florida red tide toxins } \\
\text { with increase un rates of } \\
\text { respiratory disease admissions to } \\
\text { an hospital ER (emergency Room) } \\
\text { in Sarasota, FL. } \\
\text { Admissions to an ER were as } \\
\text { compared to } 2001 \text { period were less. }\end{array}$ & {$[56]$} \\
\hline 2002 & East Timor & PSP outbreak & 1 people intoxicated and 1 death & {$[29]$} \\
\hline 2002 & South of Chile & Blooms of A.catenella & Area was closed & {$[40]$} \\
\hline $\begin{array}{l}20^{\text {th }} \\
\text { December } \\
2002 \\
\end{array}$ & $\begin{array}{l}\text { Minnie bay of Port } \\
\text { Blair in India }\end{array}$ & $\begin{array}{l}\text { Noctiluca scintillans } \\
\text { bloom }\end{array}$ & Not known & {$[57]$} \\
\hline June 2003 & $\begin{array}{l}\text { Northumberland } \\
\text { Strait (N S) }\end{array}$ & Not known & $\begin{array}{l}\text { Closure in N S due to PSP toxins } \\
\text { found in bar clams from } \\
\text { southwestern Prince Edward } \\
\text { Island. }\end{array}$ & {$[58]$} \\
\hline $\begin{array}{l}2003 \text { January } \\
\text { to March }\end{array}$ & Flordia USA & $\begin{array}{l}\text { Karenia brevis blooms } \\
\text { present along shore. }\end{array}$ & $\begin{array}{l}\text { Beach- goers exposed to } \\
\text { brevetoxins }\end{array}$ & {$[59]$} \\
\hline $\begin{array}{l}\text { September } \\
2004\end{array}$ & $\begin{array}{l}\text { South coast of } \\
\text { Thiruvananthapuram } \\
\text { in India }\end{array}$ & Noctiluca red tide & Not Known & {$[60]$} \\
\hline $\begin{array}{l}16^{\text {th }} \text { and } 17^{\text {th }} \\
\text { September } \\
2004\end{array}$ & $\begin{array}{l}\text { Kerala/ Malabar } \\
\text { coast in India }\end{array}$ & $\begin{array}{l}\text { holococcolithophore } \\
\text { blooms resulted in massive } \\
\text { fish kills } \\
\text { Cochlodinium } \\
\text { polykrikoides, Karenia } \\
\text { brevis }\end{array}$ & $\begin{array}{l}\text { A strong stench was reported and } \\
\text { around } 200 \text { children's mostly below } \\
15 \text { years of age suffered from } \\
\text { nausea, chest pain, respiratory } \\
\text { problems, and many were even } \\
\text { hospitalized. } \\
\text { About } 200 \text { hospitalized, large } \\
\text { number of fish kills }\end{array}$ & {$[46]$} \\
\hline 2005 & New England waters & $\begin{array}{l}\text { Blooms of Alexandrium } \\
\text { fundyense }\end{array}$ & Recorded levels of PSP toxins & {$[62]$. } \\
\hline 2005 & $\begin{array}{l}\text { Puget Sound, } \\
\text { Washington State }\end{array}$ & $\begin{array}{l}\text { High density blooms of } \\
\text { diatoms Pseudo-nitzschia, } \\
\text { and Domoic acid detained } \\
\text { in razor clams }\end{array}$ & $\begin{array}{l}\text { Forced closure of recreational, } \\
\text { commercial, and tribal subsistence } \\
\text { shellfish harvesting in Puget } \\
\text { Sound. }\end{array}$ & {$[63]$} \\
\hline $\begin{array}{l}\text { September } \\
2006 \text { for } 3 \\
\text { months }\end{array}$ & $\begin{array}{l}\text { Florida west Coast, } \\
\text { US }\end{array}$ & $\begin{array}{l}\text { Annual blooms of toxic } \\
\text { din flagellate Karenia } \\
\text { brevis causes red tide } \\
\text { events }\end{array}$ & $\begin{array}{l}\text { Exposure to toxic aerosols induces } \\
\text { respiratory irritation; } \\
\text { Beach Goers: eye irritation, nasal } \\
\text { congestion and dry cough. } \\
\text { Lifeguards: respiratory symptoms. } \\
\text { Coastal residents had } 54 \% \text { increase } \\
\text { in admissions in coastal hospital for } \\
\text { respiratory diagnosis (i.e. asthma, } \\
\text { pneumonia and bronchitis) during } \\
\text { onshore red tide event }\end{array}$ & {$[64]$} \\
\hline 2008 & $\begin{array}{l}\text { French Atlantic } \\
\text { Waters }\end{array}$ & $\begin{array}{l}\text { Outbreaks of } \\
\text { Chrysochromulina } \\
\text { spp. }\end{array}$ & Not known & {$[55]$} \\
\hline
\end{tabular}




\begin{tabular}{|c|c|c|c|c|}
\hline $\begin{array}{l}\text { Annual } \\
\text { occurrence }\end{array}$ & Gulf of Mexico & $\begin{array}{l}\text { Karenia brevis blooms and } \\
\text { aerosolized brevetoxins }\end{array}$ & $\begin{array}{l}\text { Respiratory irritation in humans } \\
\text { exposed at beach or boats during } \\
\text { blooms }\end{array}$ & {$[37]$} \\
\hline $\begin{array}{l}\text { Annual } \\
\text { occurrence }\end{array}$ & $\begin{array}{l}\text { US Virgin Islands and } \\
\text { French West Indies }\end{array}$ & $\begin{array}{l}\text { Species of dinoflagelletes } \\
\text { causing CFP (ciguatera } \\
\text { fish poisoning) }\end{array}$ & $\begin{array}{l}3 \% \text { of the total population per } \\
\text { year affected by CPF }\end{array}$ & {$[45,27]$} \\
\hline Not known & Florida waters & $\begin{array}{l}\text { Karenia brevis ingestion } \\
\text { of brevetoxins }\end{array}$ & $\begin{array}{l}\text { Reported brevetoxins in urine from } \\
3 \text { persons who suffered from severe } \\
\text { NSP after eating contaminated } \\
\text { shellfish from Florida } \\
\end{array}$ & {$[52]$} \\
\hline 2009 & $\begin{array}{l}\text { Washington State, } \\
2009\end{array}$ & $\begin{array}{l}\text { Akashiwo } \\
\text { sanguinea }\end{array}$ & Affected Bird ( scoters) diversity & {$[65]$} \\
\hline 2009 & Singapore & $\begin{array}{l}\text { Dinoflagellate : } \\
\text { Gambierdiscus toxicus, } \\
\text { Ostreopsis, Coolia and } \\
\text { Prorocentrum } \\
\end{array}$ & $\begin{array}{l}\text { Massive fish kill was observed for } \\
\text { the first time in Singapore waters } \\
\text { during late December 2009, killing } \\
200,000 \text { farm fish }\end{array}$ & {$[66]$} \\
\hline 2013 & Singapore & $\begin{array}{l}\text { Dinoflagellates: } \\
\text { Gambierdiscus toxicus, } \\
\text { Ostreopsis, Coolia and } \\
\text { Prorocentrum }\end{array}$ & $\begin{array}{l}\text { Around } 90,000 \mathrm{~kg} \text { of fish were found } \\
\text { dead in june } 2013 \text { in Lim Chu } \\
\text { Kang. }\end{array}$ & {$[66]$} \\
\hline 2014 & Singapore & \begin{tabular}{|l|} 
Dinoflagellates: \\
Gambierdiscus toxicus, \\
Ostreopsis, Coolia and \\
Prorocentrum \\
\end{tabular} & $\begin{array}{l}\text { Massive fish kill was observed in } \\
\text { waters during early Feb 2014, } \\
\text { killing both farm fish and wild fish. }\end{array}$ & {$[66]$} \\
\hline 2014 & Lake Erie, & $\begin{array}{l}\text { Microcystis aeruginosa. } \\
\text { Aphanizomenon spp., An } \\
\text { abaena spp., Cylindrosper } \\
\text { mopsis spp. } \\
\text { and Planktothrix spp } \\
\end{array}$ & $\begin{array}{l}\text { Three days tap water was banned } \\
\text { at Toledo, Ohio. }\end{array}$ & {$[67]$} \\
\hline 2015 & $\begin{array}{l}\text { off the Washington } \\
\text { and Oregon coast }\end{array}$ & Pseudo-nitzschia australis, & $\begin{array}{l}\text { highest levels of domoic acid } \\
\text { contamination in the food web ever } \\
\text { recorded for many species, }\end{array}$ & {$[68]$} \\
\hline 2015 & Singapore & \begin{tabular}{|l} 
Dinoflagellates: \\
Gambierdiscus toxicus, \\
Ostreopsis, Coolia and \\
Prorocentrum
\end{tabular} & $\begin{array}{l}\text { Killed } 600 \text { tones of wild and farm } \\
\text { fish in Feb } 2015\end{array}$ & {$[69]$} \\
\hline 2016 & South west Florida & Red Tide: Karenia brevis & Fish kills reported & {$[70]$} \\
\hline
\end{tabular}

The most toxic algal species are recorded among dinoflagellates but diatoms and cyanobacteria also produce some toxic chemicals. Major classes of toxins produced by cyanobacteria include hepatotoxins (microcystins, nodularin), cytotoxin (cylindrospermopsin), neurotoxins (saxitoxins, anatoxins) and endotoxins (lypopolysaccharides). The hepatotoxic and neurotoxic metabolites of cyanobacteria are hazardous to humans and animals and accumulate in the liver and the kidney of the fish[71]. The toxins implicated in human illness are microcystins, cylindrospermopsin, anatoxins, saxitoxins, and methylamino alanine. Among the cyanotoxins, microcystins and cylindrospermopsins are more commonly produced by cyanobacteria.

Some of the crucial toxins produced by diatoms and dinoflagellates and their syndromes are described below:

\subsubsection{Amnesic Shellfish Poisoning (ASP)/ Domoic Acid Poisoning (DAP)}

1. Causative organism: Diatom Pseudo-nitzschia

2. Species: Pseudo-nitzschia australis \& Psedo-nitzschia multiseries[13]

3. Toxins produced: Domoic acid

4. Toxins transferable through: clams oysters, crabs, anchovies, and sardines[72]

ASP is also called as DAP (Domoic Acid Poisoning) as these planktonic pinnate diatoms produce DA 
(domoic acid) that causes ASP. Amnesic shellfish poisoning causes gastrointestinal and neurological disorders. Consumption of affected shellfish may lead to gastroenteritis showing symptoms of nausea, vomiting, diarrhoea, and abdominal cramps within 24 hours. Neurological symptoms like dizziness; headache, seizures, respiratory problems, short-term memory loss and coma usually appear within 48 hours of consumption of toxic shellfish. In the year 1987, four Canadians lost their lives when exposed to toxic mussels from Prince Edward Island, Canada. Along the west coast of the U.S in the year 1991, about 100 brown pelicans and cormorants in Monterey Bay died or suffered from neurological disorders. It was later identified that blooms of diatom Pseudo-nitzschia australis caused ASP. In the same year, in the month of October and November, DA was found in razor clams (Siliqua patula Dixon) and Dungeness crabs (Cancer magister Dana) on the Oregon and Washington Coasts. During the periods of 1991 and 1994, blooms of $P$. australis most commonly observed in US remained for a longer period of time until late summer and autumn. In the year 1994, blooms of pseudo-nitzchia sp. mainly $P$. multiseries, $P$. australis and P. pungens persisted for more than 6 weeks in the Hood Canal of Western Washington.

According to Stewart et. al.[73], DA is metabolized by bacteria of genera Alteromona and pseudomonas, present in Blue mussels (M. edulis). Scallops are reported not to contain these toxic DA. On the other hand O'Shea[74], reports that the Sea Otter is the only one animal known to avoid intoxication, probably recognizing toxic prey by their odour. Scholin et al.[75], as cited in Rue and Bruland[76], observed mass mortality of California sea lions in Monterey Bay due to blooms of Pseudonitzschia australis. Further, Rue et. al. [76] concluded that DA produced by species of Pseudonitzschia could bind iron and copper causing mortality of about 400 sea lions between the months of May and June 1998.

\subsubsection{Paralytic Shellfish Poisoning (PSP)}

1. Causative organism: Dinoflagellates like Alexandrium spp., Gymnodium catenatum and Pyrodinium bahamense[72]

2. Species: A. acatenella, A. catenella, A. hiranoi, A ostenfeldii and A. tamrense[13]

3. Toxins produced: saxitoxins (STX), neosaxitoxin (NEO), gonyautoxins (GTXs) and C-toxins. $[77]$

4. Toxins transferable through: saxitoxins accumulation in mussels, clams, oysters and fishes like sardines, herring and puffer fish (without harming them) $[72]$

Paralytic shellfish poisoning is caused due to a group of marine phycotoxins produced by several dinoflagellates. These toxins get accumulated in shellfish, and when consumed by vertebrates, block sodium channels leading to paralysis attack. Associated symptoms are mostly neurological. Other symptoms include numbness, giddiness, tingling, burning of perioral region, ataxia, drowsiness, fever, rash and staggering. Within 5 to 30 minutes of consumption, slight perioral tingling is observed progressing to numbness that spreads to the face and neck in moderate cases and further if medical support is not available within 24 hours it can cause complete paralysis leading to a severe respiratory attack.

In 1976, Malaysia was subjected to toxic blooms of Pyrodinium bahamense var. compressum, affecting 202 people and causing death of 7 children. In 2002, blooms of A. catenella had hit south of Chile to such an extent that the President declared closure of this area[40].In India incidences of PSP are reported along the east coast as well as along the west coast.

\subsubsection{Diarrhetic Shellfish Poisoning (DSP)}

1. Causative organism: Dinoflagellates like Dinophysis spp., Prorocentrum spp.,

2. Species: D. acuminata, D. acuta, D. fortii, D. norvegica, [13] and D. caudate [48]

3. Toxins produced: Okaidic acid

4. Toxins transferable through: mussels, scallops, clam etc.

DSP causes gastrointestinal illness without neurological clams disorders. Gastrointestinal symptoms include diarrhoea, nausea, vomiting, abdominal cramps and chills within 30 min to 12 hrs from ingestion. However recovery can occur within 3 days of medical treatment. DSP is caused by certain species of dinoflagellates. The first reported outbreak of DSP took place in the 1960s in the Netherlands, followed by Japan in 1970s. Since then many cases have been reported from several parts of world, including Europe, South America, North America Asia Australia and New Zealand (Mak et. al., 2005)[78]. About 500 cases of DSP were reported in Spain in 1981 with 2000 cases in France in the years 1984 and 1986. In 1990, mussels exported from Denmark to France caused DSP poisoning in over 400 people. 
Karunasagar et al. [48] observed species of D. caudate causing DSP in infected shellfishes collected from the harvesting areas along the coast of Karnataka in India.

\subsubsection{Ciguatera Fish Poisoning (CFP)}

1. Causative organism: Dinoflagellates like Gambierdiscus toxicus, Prorocentrum sp., Ostreopsis spp., Coolia monotis, Thecadinium sp. and Amphidinium sp.

2. Species: Amphidinium carterae

3. Toxins produced: Ciguatoxins / maitotoxin

4. Toxins transferable through: small-algae eating fish (e.g. reef fish) and passed up to food chain to predators such as barracuda, grouper snapper, jacks and kingfish (without harming)[72]

CFS causes neurological symptoms like paresthesias, pain in the teeth, pain on urination, blurred vision, temperature reversal, gastrointestinal effects like that of diarrhoea, abdominal cramps and vomiting, and cardio-vascular symptoms like heart block and arrhythmias. All these symptoms are likely to occur within few hours of ingestion. Ciguatera can also be transmitted sexually. Exposure of any pregnant woman to it may lead to premature labour and spontaneous abortion, and effects on the foetus and newborn child through placental and breast milk transmission have been reported. Reports from the US Virgin Islands observe that 3\% of the total population per year is affected. This is similar to that of the French West Indies.

\subsubsection{Neurotoxic Shellfish Poisoning (NSP)}

1. Causative organism: Dinoflagellates like Gymnodinium spp. $[72,75]$

2. Species: Kerenia brevis (formerly known as Gymnodinium breve)

3. Toxins produced: Brevetoxins

4. Toxins transferable through: accumulates in shellfishes, sea grasses (without harming)[72]

NSP produces symptoms identical to that of CFP, which includes gastrointestinal, and neurological symptoms. In addition to this, it produces asthma-like symptoms through aerosols. It also causes massive fish kills and other respiratory problems. Airborne toxins may cause irritations in eyes, nose, and throat and cause sinus infection. It poisons manatees, dolphins, sea turtles and sea birds as well as humans on inhalation or through contaminated seafood. The causative species of Kerenia brevis produces 2 types of lipid soluble toxins which are hemolytic and neurotoxic, out of which neurotoxic toxins are known as brevetoxin.

Since its recognition as the cause of massive fish kills and initial description in 1948)[79], blooms of Karenia brevis have been documented almost annually along Florida's southwest coastal region[37]. Cases have been reported in other coastal regions of the Gulf from Alabama to Texas and the eastern coast of Mexico ([28]as well as along the southeast Atlantic coast to North Carolina[80]). The commercial shellfish industry experienced repeated and prolonged shellfish bed closures due to the presence of K. brevis throughout the year during the severe Karenia bloom of 2005 in the eastern Gulf of Mexico[30].

\section{Control of Algal Blooms}

Control of algal blooms is crucial to maintain health of coastal and marine ecosystems. Reducing nutrient loads from various land based activities into coastal systems will help in control of algal blooms. Managing nutrient inputs is the critical factor for managing algal blooms. However, this is not the sole approach for management of algal blooms. This section presents some approaches for control of algal blooms.

\subsection{Control of HABs by Managing Nutrient Inputs}

Martins et al[81] suggest that reductions in nitrogen loading should, in many cases, be geared to reductions in phosphorus, so that $\mathrm{N}$ : P loading ratios are kept sufficiently high to discourage N2-fixing cyanobacteria. Further, coastal marine simulation models by Kiirikki et al. [82] suggest that phosphorus removal at St. Petersburg, Russia, would reduce the biomass of nitrogen-fixing cyanobacteria in the entire Gulf of Finland. Similarly, Schernewski and Neumann[83] observe that reducing phosphorus loads in the most polluted rivers could be the most cost-effective way of improving water quality in the Baltic Sea. Thus, a high N: P ratio in estuaries or riverine waters may be the key factor in controlling HABs in 
coastal and marine ecosystems.

\subsection{Control of HABs Through Biomanipulating}

The possibility of controlling the symptoms of eutrophication in North American lakes by 'biomanipulating' higher members of aquatic food chains instead of controlling nutrient inputs was pioneered by Joseph Shapiro and his students[80,84,85]. They suggest that eutrophication can be intensified by the removal of piscivorous predators such as bass, pike and walleye by overfishing. This causes an increase in zooplanktivores, which in turn reduce grazing herbivores to low levels, allowing phytoplankton to flourish. Thus removal of piscivorous predators could cause a lake to move from a low algal phase to a high algal phase at the same nutrient loading. Similar results have been demonstrated by Carpenter and Kitchell[86] and Elser et al.[87]. Further, some grazers may increase the supply of nitrogen relative to phosphorus, by selective excretion of nitrogen $[87,88]$. The high $\mathrm{N}$ : $\mathrm{P}$ ratio in grazerexcreted nutrients in some systems thus can allow nitrogen-fixing cyanobacteria to be outcompeted by other species.

\subsection{Control of HABs through Chemical Treatment}

It is observed that internal regeneration of nutrients may hinder recovery from eutrophication. This hindrance may correspond to the iron concentration in the system. Though the mechanism is not yet fully known, a possible reason for this phenomenon is that in high-iron systems, phosphorus coprecipitates with ferric hydroxide when oxygen is abundant in the water column. If iron concentration is low, phosphorus is free from control by iron coprecipitation, and is free to diffuse into overlying water, where it facilitates increased algal growth[81]. Recycling of phosphorus from sediments can be avoided by treatment with iron or alum. It is found that addition of iron or alum can prevent the internal recycling of phosphorus and thus maintain a higher $\mathrm{N}$ : $\mathrm{P}$ ratio, which, in turn, may control HABs.

\section{Conclusions}

The increasing occurrence of toxic algal blooms has been reported worldwide in the past few decades. These increasing incidents of algal blooms are contributed by various causes such as eutrophication of the coastal areas, dust storms and metals, climate change and increase in temperature, upwelling of oceans, ballast water from ships, unhealthy coral reefs etc. The human ingestion of marine organisms contaminated with algal toxins provokes pathological symptoms termed biopoisonings, and may even lead to high levels of mortality. Hence, there is an urgent need for control of harmful algal blooms. Reductions in phosphorus inputs in estuarine or riverine waters may be the key factor in controlling HABs in coastal and marine ecosystems.

Acknowledgements. Part of an earlier version of this paper was an output of the project 'Harmful algal blooms along the West Coast of India for preparedness in the event of a bloom' financially supported by the Council of Scientific and Industrial Research (CSIR), India by way of research grant to Dr Sangeeta Sonak. Authors gratefully acknowledge financial support from the CSIR for carrying out this work.

\section{References}

1. Chattopadhyay, J., Sarkar, R.R., Pal. S., Mathematical modelling of harmful algal blooms supported by experimental findings. Ecological Complexity, 2004, 1, 225-235.

2. Van den Bergh, J.C.J.M., Nunes, P.A. L.D., Dotinga, H.M., Kooistra, W.H.C.F., Vrieling, E. G., Peperzak. L., Exotic harmful algae in marine ecosystems: an integrated biological - economic - legal analysis of impacts and policies. Marine Policy, 2002, 26, 59-74

3. Fleming, L. E., Easom, J., Baden, D., Rowan, A., Levin, B., Emerging harmful algal blooms and human health: Pfiesteria and related organisms. Toxicol Patho., 1999, 27, 573 - 581. 
4. Ulèn, B.M., Weyhenmeyer, G.A., Adapting regional eutrophication targets for surface waters-influence of the EU Water Framework Directive, national policy and climate change. Environmental Science and policy, 2007,10,734-742.

5. Onderka, M., Correlations between several environmental factors affecting the bloom events of cyanobacteria in Liptovska Mara reservoir (Slovakia) - A simple regression model. Ecological Modelling, 2007, 209, 412-416.

6. O'Neil J. M., Davis T. W., Burford M A., Gobler C. J. (2012) The rise of harmful cyanobacteria blooms: The potential roles of eutrophication and climate change Harmful Algae 2012, 14, 313-334

7. Chapra, S.C., Surface Water-quality Modeling, The McGraw-Hill, New York, 1997, p. 526.

8. Zingone, A. and Enevoldsen, H.O, The diversity of harmful algal blooms: challenge for science and management. Ocean and Coastal Management, 2000, 43,725-748.

9. Preece E. P., Hardy F. J., Moore B. C., Bryan M., A review of microcystin detections in Estuarine and Marine waters: Environmental implications and human health risk, Harmful Algae, 2017, 61, 31-45.

10.Smith, V.H., Low nitrogen to phosphorus ratios favor dominance by blue-green algae in lake phytoplankton, Science, 1983, 221,669-671.

11.Bulgakov, N. G., Levich, A. P., The nitrogen: phosphorus ratio as a factor regulating phytoplankton community structure. Arch. Hydrobiol., 1999, 146, 3-22.

12.Lukatelich, R.J., McComb, A.J., Nutrient levels and the development of diatom and blue-green algal blooms in a shallow Australian estuary. J. Plankton Res., 1986, 8, 597-618.

13.Horner, R. A., Garrison, D.L., Plumley, F.G., Harmful algal blooms and red tide problems on the U.S. west coast. Limnol Oceanography, 1997, 42, 1076 - 1088.

14.Silbergeld, E. K., Grattan L, Oidach, D., Morris, J.G., Pfiesteria: harmful algal blooms as Indicator of Human: Ecosystem interactions. Environmental Research Section A, 2000, 82, 97-105.

15.Garcia-Hernandez, J., Garcia-Rica, L., Jara-Marini, E. E., Barraza-Guaradado, R., Weaver, A. H., Concentrations of heavy metal in sediments and organism during a harmful algal bloom (HAB) at Kun Kaak Bay, Sonora, Mexico. Marine Pollution Bulleti., 2005, 50, 733-739.

16.Semeneh, M., Dehairs, F., Elskens, M., Baumann, M.E.M., Kopezynska, E.E., Lancelot, C., Goeyens, L., Nitrogen uptake regime and phytoplankton community structure in the Atlantic and Indian Sectors of the southern ocean. Journal of Marine Systems, 1998, 17, 159-177.

17.News Report number PII: S2225-326X (01) 00229-6. Red tides and dust storms. Marine Pollution Bulletin, 2001, 42, p. 796.

18.Albert, S., O’Neil, M. J., Udy, J.W., Ahern, S. K., O'Sullivan, C. M., and Dennison, W.C., Blooms of the cyanobacterium Lyngbya majuscula in coastal Queensland, Australia: disparate sites common factors. Marine Pollution Bulletin, 2005, 51, 428-437.

19.Gobler, C. J., Donat, J. R., Consolvo, IIIb. J. A, Sanudo-wilhemy, S.A., Physicochemical speciation of iron during coastal algal blooms. Marine Chemistry, 2002, 77, 71-89.

20.Peperzak, L., Climate change and harmful algal blooms in the North Sea. Acta. Oecologica, 2003, 24, S139-S144.

21.Sharma, G.S., Upwelling off the Southwest coast of India. Indian Journal of Marine Sciences, 1978, 7, 209-218.

22.Ramana ,T.V., Reddy, M.P.M., Upwelling and sinking in the Arabian sea along Dakshina Kannada coast. Environment \& Ecology, 2006, 24, 379-384.

23.Anil, A.C., Venkat, K., Sawant, S. S., Dileepkumar. M., Dhargalkar, V. K., Ramaiah, N., Harkantra, S. N., and Ansari, Z. A., Marine bioinvasion: concern for ecology and shipping. Current Science, 2002, 83, 214-218.

24.Untawale, A.G., Agadi, V.V., Dhargalkar, V.K., Mahasagar: Bulletin National Institute of Oceanography, 1980, 23, 179-181.

25.Waldichuk, M., Marine biotoxins and human activity. Marine Pollution Bulletin, 1990, 21, 215-216.

26.Carmichael W. W., Boyer G. L., Health impacts from cyanobacteria harmful algae blooms: Implications for the North American Great Lakes Harmful Algae, 2016, 54, 194-212

27.HARRNESS Harmful Algal Research and Response: A National Environmental Science Strategy 2005 - 2015 (eds Ramsdell, J. S., Anderson, D.M., Glibert, P.M.,) Ecological Society of America, Washington DC, 2005, p. 96. 28.Magaña, H.A., Contreras, C., Villareal, T. A., A historical assessment of Karenia brevis in the western Gulf of Mexico. Harmful Algae, 2003, 3, 163-171.

29.Batorèu, M. C. C., Dias, E., Pereira, P., Franca, S., Risk of human exposure to paralytic toxins of algal origin. Environmental Toxicology and Pharmacology, 2005, 19, 401-406. 
30.Taylor, H.F., Mortality of fishes on the west coast of Florida. Report of the U. S., Commission on Fisheries Document, 1917, 848, 1-24.

31.Akiba, T., Hattori Y., Food poisoning caused by eating asari and oyster-toxic substance, venerupin. Japanese Journal Experimental Medicine, 1949, 20, 271-284.

32.Nakajima, M. Studies on the source of shellfish poison in Lake Hamana. I. Relation of the abundance of a species of dinoflagellate, Prorocentrum sp. to shellfish toxicity. Bulletin. Japanese Society Scientific Fishery, 1965 a, 31 , 198-203.

33.Nakajima. M., Studies on the source of shellfish poison in Lake Hamana. II. Shellfish toxicity during the 'redtide'. Bulletin. Japanese Society Scientific Fishery, 1965 b, 31, 204-207.

34.Nakajima, M., Studies on the source of shellfish poison in Lake Hamana. III. Poisonous effects of shellfish feeding on Prorocentrum sp. Bulletin. Japanese Society Scientific Fishery, 1965 c, 31, 281-285.

35.Nakajima, M., Studies on the source of shellfish poison in Lake Hamana. IV. Identification and collection of the noxious dinoflagellates. Bulletin. Japanese Society Scientific Fishery, 1968, 34, 130-131.

36.Woodcock, A.H., Note concerning human respiratory irritation associated with high concentrations of plankton and mass mortality of marine organisms. Journal of Marine Research, 1948, 7, 56- 62.

37.Heil, C.A., Steidinger, K. A., Monitoring, management, and mitigation of Karenia blooms in the eastern Gulf of Mexico. Harmful Algae, 2009, 8, 611-617

38.Backer, L. C., Impacts of Florida red tides on coastal communities. Harmful Algae, 2009, 8, 618-622.

39.Music, S.I., Howell, J.T., Brumback, L.C., Red tide: its public health implications. Journal of the Florida Medical Association, 1973, 60, 27-29.

40.Bhat, S.R. and Matondkar, S.G.P., Algal blooms in the seas around India - networking for research and outreach. Current Science, 2004, 87, 1079 - 1083.

41.Nayak, B.B., Karunasagar, I., Karunasagar, I., Bacteriological and physio-chemical factors associated with Noctiluca miliaris bloom, along Mangalore, southwest coast of India. Indian Journal of Marine Sciences. 2000, 29,139-143.

42.Venugopal, P., Harida, P., Madhupratap, M., Rap, T.S.S., Incidence of red water along South Kerala coast. Indian Journal of Marine Sciences, 1979, 8, 94-97

43.Tangen, K., Shellfish poisoning and the occurrence of potentially toxic dinoflagellates in Norwegian waters. Sarsia, 1983, 68, 1-7.

44.Langeland, G., Hasselgaard, T., Tangen, K., Skulberg, O.M., Hjelle, A., An outbreak of paralytic shellfish poisoning in western Norway. Sarsia, 1984, 69, 185-193.

45.http://www.whoi.edu/redtide/.

46.Bhat, S.R., Devi, P., D'Souza, L., Verlecar, X. N., Naik, C. G., Harmful algal blooms. In Multiple dimensions of global environmental change (eds Sonak, S.), TERI Press, New Delhi, 2006, pp. 419-431.

47.Sombrito, E. Z., Bulos, A. M., Sta, Maria, E. J., Honrado, M.C.V., Azanza, R.V., Furio E, F., Application of 210Pb-derived sedimentation rates and dinoflagellate cyst analyses in understanding Pyrodinium bahamense harmful algal blooms in Manila Bay and Malampaya Sound, Philippines. Journal of Environmental Radioactivity, 2004, 76, 177-194.

48.Karunasagar, I., Segar, K., Karunasagar, I., Potentially Toxic Dinoflagellates in shellfish harvesting areas along the coast of Karnataka state (India). In Red Tides ( eds Okaichi, T., Aderson, M. D., Nemoto, T., ) Elsevier Science Publishing Co., New York, 1989, pp. 65-68.

49.Freudenthal, A. R. and Jijina, J., Shellfish poisoning episodes involving or coincidental with dinoflagellates. In Toxic Dinoflagellates (eds Anderson, D.M., White, A. W, and Baden, D.G.), Elsevier, New York, 1985, pp. 461466.

50.Morris, P., Campbell, D.S., Taylor, T.J., Freeman, J.I., Clinical and epidemiological features of neurotoxic shellfish poisoning in North Carolina. American Journal of Public Health. 1991, 81, 471-473. \

51.Watkins, S.M., Reich, A., Fleming, L.E., Hammond, R., Neurotoxic shellfish poisoning. Marine Drugs, 2008, 6, 431-455.

52.Kirkpatrick, B., Fleming, L.E., Squicciarini, D., Backer, L.C., Clark, R., Abraham, W., Benson, J., Chenge, Y.S., Johnson, D., Pierce, R., Zaias, J., Bossart, G. D., Baden, D. G., Literature review of Florida red tide: implications for human health effects Barbara. Harmful Algae, 2004,3, 99-115. 
53.Shanmugam, P., Ahn,Y., Prakya, S.R., SeaWiFS .Sensing of hazardous algal blooms and their underlying mechanisms in shelf-slope waters of the Northwest Pacific during summer. Remote Sensing of Environment, 2008, $112,3248-3270$

54.Backer, L.C., Fleming, L. E., Rowan, A., Cheng, Y., Benson, J., Pierce, R. H., Zaias, J., Bean, J., Bossart, G.D., Johnson, D., Quimbo, R., Baden, D.G., Recreational exposure to aerosolized brevetoxins during Florida red tide events. Harmful Algae, 2003, 2, 19-28.

55.http://ioc-unesco.org/hab/index.php?option=com_oe\&task=viewDoclistRecord\&doclistID=59 Last accessed on $26 / 06 / 09$.

56.Kirkpatrick, B., Fleming, L.E., Backer, L.C., Bean, J.A., Tamer, R., Kirkpatrick, G., Kane, T., Wanner, A., Dalpra, D., Reich, A., Baden, D.G., Environmental exposures to Florida red tides: Effects on emergency room respiratory diagnoses admissions. Harmful Algae, 2006, 5, 526-533.

57.Dharani, G., Nazar, A. A. K., Kanagu. L., Venkateshwaran, P., Kumar, T.S., Ratnam, K., Venkatesan, R., Ravendran, M., On the recurrence of Noctiluca Scintillans blooms in Minnie Bay, Port Blair: Impact on Water quality and bioactivity of extracts. Current Science, 2004, 87, 990-994.

58.http://www.glf.dfo-mpo.gc.ca/os/northumberland/si-is/summary-algal_sommaire-algues-e.php\#top.

59.Pierce, R. H., Henry, M.S., Blum, P.C., Hamel, S.L, Kirkpatrick, B., Cheng, Y.S., Zhou, Y., Irvin, C.M., Naar, J., Weidner, A., Fleming, L.E., Backer, L.C., Baden, D.G., Brevetoxin composition in water and marine aerosol along a Florida beach: Assessing potential human exposure to marine biotoxins. Harmful Algae, 2005, 4, $965-972$.

60.Sahayak, S., Joythibabu ,R., Jayalakshmi, K.J., Habeebreeman, H., Sabu, P., Prabhakaran M, P., Jasmine, P., Shaiju, P., Rejomon, G., Thiresiamma, J., Nair, K., K. C., Red tide of Noctiluca Miliaris off south of Thiruvananthapuram subsequent to the stench event at the southern Kerala coast. Current science, 2005, 89,1472-1473.

61.Ramaiah, N., Paul, J.T. Fernandes, V., Raveendran, T., Raveendran, O., Sundar, D., Revishandran, C., Shenoy, D.M., Mangesh, G., Kurian, S., Gerson, V.J., Shoh, D.T., Madu, N.V., Kumar, S.S., Lokabharathi, P.A., Shetye, S.R., The September 2004 stench off the southern Malabar coast - A consequence of Holoccolithophore bloom. Current science, 2005, 88, 551-554.

62.Jin, D., Hoagland, P., The value of harmful algal bloom predictions to the nearshore commercial shellfish fishery in the Gulf of Maine. Harmful Algae, 2008, 7, 772-781.

63.Trainer, V.L., Cochlan, W.P., Erickson, A., Bill, B.D., Cox, F.H., Borchert, J.A., Lefebvre, K. A ., Recent domoic acid closures of shellfish harvest areas in Washington State inland waterways. Harmful Algae, 2007, 6, 449-459.

64.Kirkpatrick, B., Currier, R., Nierenberg, K., Reich, A., Backer, L.C., Stumpf, R., Fleming, L., Kirkpatrick, G., Florida red tide and human health: a pilot beach conditions reporting system to minimize human exposure. Science of the total environment, 2008, 402, 1-8.

65.Kudela, R.,Overview of Harmful Algal Blooms: A Global Perspective.2016. Available online at https://www.epa.gov/sites/production/files/2016-03/documents/overview-habs-global-perspective.pdf

66.Leon, S. C. Y., Introduction to Bloom Species in Singapore and its vicinity.2015.Available online at http://iocwestpac.org/files/upload_manual/Lecture2_BlmSpecies_Sandric.pdf

67.Hoa, J. C., Michalak A. M., Challenges in tracking harmful algal blooms: A synthesis of evidence from Lake Erie. Journal of Great Lake Research, 2015, 41, 317-325. http://dx.doi.org/10.1016/j.jglr.2015.01.001

68.Hickey, H.,Ocean conditions contributed to unprecedented 2015 toxic algal bloom. Available online at http://www.ocean.washington.edu/story/2015_Toxic_Algal_Bloom

69.Lim, L. P. and Leong, S., Harmful Algal Blooms in Singapore Coastal Waters.2015 Available online at: https://www.tmsi.nus.edu.sg/files/Lim\%20Lay\%20Peng_HABs\%20in\%20Singapore.pdf

70.Red Tide status report south west coast Florida. 2016. http://www.bradenton.com/latestnews/article71114842.ece/BINARY/Red\%20tide\%20in\%20southwest\%20Florida,\%20April\%208,\%202016.

71.Wiegand, C. and Pflugmacher, S., Ecotoxicological effects of selected cyanobacterial secondary metabolites: a short review. Toxicology and Applied Pharmacology, 2005, 203, 201-18.

72.Weiss, K.R., Paying a price for paradise: Sea breeze carries arsenal of toxins ashore during red tide outbreaks. The Baltimoresun.com, From the Los Angeles Times, third of five parts, 2006. Available online at: http://www.baltimoresun.com/news/health/balte.oceans01aug01,0,7230859.story?coll=bal-health-headlines . 
73.Stewart, J.E., Marks, L.J., Gilgan, M.W., Pfeiffer, E., Zwicker, B.M., Microbial Utilization of the neurotoxin domoic acid:blue mussels (Mytilus edulis and soft clams (Mya arenaria) as sources of the microorganisms. Canadian Journal of Microbiology, 1998, 44, 456-464.

74.O'shea, T., Environmental Contaminants and Marine Mammals. In Biology of Marine Mammals (eds Reynolds, J.E. and Rommel, S.A), Smithsion Institution Press, Washington, D. C. 1999.

75.Scholin, C.A., Gulland, F., Doucette, G.J., Benson, S., Busman, M., Chavez, F.P., Cordaro, J., DeLong, R., DeVogelaere, A., Harvey, J., Haulena, M., Lefebvre, K., Lipscomb, T., Loscutoff ,S., Lowenstine, L.J., Marin III, R., Miller, P.E., McLellan, W.A., Moeller, P.D.R., Christine, Powell CL, Rowles, T., Silvagni, P., Silver, M., Spraker, T., Trainer, V., Van Dolah, F.M. Mortality of sea lions along the central California coast linked to a toxic diatom bloom. Nature, 2000, 403, 80-84.

76.Rue, E., Bruland, K., Domoic acid binds iron and copper: a possible role for the toxin produced by the marine diatom Pseudo-nitzschia. Marine Chemistry, 2001, 76, 127-134.

77.Gubbins, M. J., Eddy, F. B., Gallacher, S., Stagg, R. M., Paralytic shellfish poisoning toxins induce xenobiotic metabolizing enzymes in Atlantic salmon (Salmo Salar). Marine Environmental Research, 2000, 50, 479-483.

78.Mak., K.Y.C., Yu, H., Choi, M.C., Shen, X., Lam, M. W. H. , Marin, M., Wu, R.S.S., Wong, P. S., Richardson, B.J., Lam, P.S.K., Okadiac acid, a causative toxin of diarrhetic shellfish poisoning in green-lipped mussels Perna Viridis from Hong Kong Fish culture zones: Method and development and monitoring. Marine Pollution Bulletin, 2005, 51, 1010-1017.

79.Davis, C.C., Gymnodinium brevis sp. nov., a cause of discolored water and animal mortality in the Gulf of Mexico. Botanical Gazette, 1948, 109, 358-360.

80.Tester, P. A., and Steidinger, K. A., Gymnodinium breve red tide blooms: Initiation, transport, and consequences of surface circulation. Limnol. Oceanogr. 1997,42, 1039-1051.

81.Martins, J. C., Lea ̃, P. N., Vasconcelos V Differential protein expression in Corbicula fluminea upon exposure to a Microcystis aeruginosa toxic strain. Toxicon, 2009, 53, 409-416.

82.Kiirikki, M., et al., Evaluating the effects of nutrient load reductions on the biomass of toxic nitrogen-fixing cyanobacteria in the Gulf of Finland, Baltic Sea, Boreal. Environmental Research, 2001, 6, 131-146.

83.Schernewski, G., Neumann, T., Perspectives on eutrophication abatement in the Baltic Sea. In Littoral 2002: The Changing Coast, 2, pp. 503-511. Eurocoast/ EUCC, 2002

84.Shapiro, J., Wright, D. I., Lake restoration by biomanipulation: Round Lake, Minnesota-the first two years. Freshw. Biol., 1984, 14, 371-383.

85.Shapiro, J., et. al., Biomanipulation: an ecosystem approach to lake restoration. In Water Quality Management through Biological Control (eds Brezonik, P.L. and Fox, J.L.), University of Florida, 1975, pp. 85-96.

86.Carpenter, S.R., Kitchell, J.F., The Trophic Cascade in Lakes, Cambridge University Press, 1993.

87.Elser, J. J. et al., Pelagic C: N: P stoichiometry in a eutrophied lake: responses to a whole-lake food-web manipulation. Ecosystems, 2000, 3, 293-307.

88.Scheffer, M., et. al., Alternative equilibria in shallow lakes. Trends Ecol. Evol., 1993, 8, 275-279. 